\title{
LE HANDICAP MONTAGNE ET SES INCIDENCES SUR LA GESTION ET L'EXPLOITATION FORESTIĖRES : LE CAS DE LA MAURIENNE (SAVOIE, FRANCE)
}

\author{
E. CONSTANTIN - P. VAUTERIN
}

Depuis le milieu des années 80 , beaucoup d'acteurs économiques de la forêt de montagne (propriétaires, gestionnaires, exploitants) parlent du "handicap" de cette forêt et réclament des aides spécifiques en s'appuyant sur la grave crise que connaît le secteur. Un modèle d'aide fréquemment évoqué est celui de l'indemnité spéciale de montagne accordée aux exploitants agricoles de montagne, pour lesquels ce soutien public est le reflet de "l'expression de la solidarité nationale, en compensation des handicaps naturels et de la prise en compte des spécificités montagnardes". Pourquoi la filière forêt-bois, qui participe également à l'aménagement du territoire en montagne, ne profiterait-elle pas de la même solidarité nationale?

De fait, les acteurs de la filière ont tous de bonnes raisons à faire valoir pour justifier leurs demandes de soutien à la forêt de montagne. Le propriétaire met en avant le concept d'effet de sillage : le bon fonctionnement de la fonction de production doit, selon lui, permettre d'assurer automatiquement la satisfaction des autres besoins écologiques, patrimoniaux, paysagers. II met ainsi en garde contre la déprise sylvicole qui peut se révéler catastrophique pour le rôle de protection et pour la capacité d'accueil de la forêt. Le gestionnaire, quant à lui, rappelle qu'il ne peut y avoir dans nos forêts fortement artificialisées - beaucoup de celles-ci datent des reboisements du siècle dernier - de gestion durable sans exploitation. Ceci est particulièrement le cas des peuplements irréguliers où, là encore plus qu'ailleurs, l'exploitation représente la principale opération sylvicole. L'exploitantscieur et le transformateur font enfin remarquer que la filière forêt-bois est un gisement d'emploi rural particulièrement intéressant dans des zones où l'emploi industriel est souvent soit traditionnellement faible, soit en fort déclin. Ainsi, en région Rhône-Alpes, l'exploitation et le traitement jusqu'à la première transformation de $300 \mathrm{~m}^{3}$ de bois permettraient de créer un emploi pour un an (SERFOB Rhône-Alpes).

Si la compétition inter-matériaux est vive, une concurrence existe au sein d'une même matière première entre les zones d'approvisionnement. Compte tenu des contraintes d'approvisionnement des centres de première transformation, les bois de montagne sont-ils aussi compétitifs que ceux de plaine ? Sinon, comment quantifier ce handicap? 


\section{CONTRAINTES ET AVANTAGES DE LA FORÊT DE MONTAGNE}

\section{Caractéristiques de la forêt de montagne}

La forêt de montagne est définie par l'Inventaire forestier national comme située à une altitude dépassant 600 à $1200 \mathrm{~m}$ suivant les régions et les expositions. La limite supérieure de cette forêt peut monter selon les massifs au maximum à $2300 \mathrm{~m}$.

Elle couvre une surface de 4,25 millions d'hectares et se répartit suivant sept grands massifs avec un taux moyen de boisement de $41 \%$ : Massif Central, Alpes du Nord, Alpes du Sud, Pyrénées, Vosges, Jura et Corse. En raison de cette grande extension géographique, elle peut présenter des situations très diverses (Barthod et Pignard, 1995). Cependant, elle présente également des caractéristiques propres dont la combinaison permet de la définir :

- L'existence de pentes fortes et continues : la longueur des pentes, qui représente la principale difficulté, est caractéristique de la montagne.

Tableau I

Répartition de la forêt publique suivant la pente (données IFN)

\begin{tabular}{|c|c|c|}
\hline Zones géographiques & $\begin{array}{l}\text { Pourcentage de surface } \\
\text { dont la pente est }<30 \%\end{array}$ & $\begin{array}{l}\text { Pourcentage de surface } \\
\text { dont la pente est }>30 \%\end{array}$ \\
\hline Plaines et collines $\ldots \ldots \ldots \ldots \ldots \ldots \ldots \ldots$ & 93 & 7 \\
\hline Alpes du Nord $\ldots \ldots \ldots \ldots \ldots \ldots \ldots \ldots$ & 23 & 77 \\
\hline Alpes du Sud & 19 & 81 \\
\hline Montagnes de Corse & 25 & 75 \\
\hline Jura $\ldots \ldots \ldots \ldots \ldots \ldots \ldots \ldots \ldots \ldots \ldots \ldots$ & 66 & 34 \\
\hline 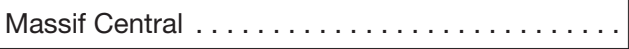 & 61 & 39 \\
\hline Pyrénées $\ldots \ldots \ldots \ldots \ldots \ldots \ldots \ldots \ldots \ldots$ & 18 & 82 \\
\hline Vosges .... & 43 & 57 \\
\hline Ensemble des montagnes $\ldots \ldots \ldots \ldots \ldots$ & 35 & 65 \\
\hline Zone méditerranéenne $\ldots \ldots \ldots \ldots$ & 56 & 44 \\
\hline
\end{tabular}

- La présence de nombreux obstacles (escarpements, blocs rocheux, falaises,...).

- Des conditions climatiques difficiles avec notamment une température plus basse: moins $0,55^{\circ} \mathrm{C}$ par tranche d'altitude de $100 \mathrm{~m}$; un enneigement prolongé : en moyenne trois mois à $600 \mathrm{~m}$, cinq mois à $1300 \mathrm{~m}$ et sept mois à $1800 \mathrm{~m}$; des vents plus violents; une durée de la période de végétation plus courte : huit mois à $1000 \mathrm{~m}$, six mois à $1500 \mathrm{~m}$ (Rameau, Mansion et Dumé, 1993) ; une exposition importante au vent.

- Une très grande hétérogénéité stationnelle (variation suivant le stade d'évolution du sol, l'exposition, etc.).

- Un rôle majeur de protection physique (contre les avalanches, les éboulements, les crues ou laves torrentielles, etc.) ; I'ONF estime que le quart environ des 400000 ha de forêt de Savoie, Haute-Savoie et Isère joue un rôle effectif de protection. En moyenne, des études ont montré que le débit de pointe de crue d'un bassin boisé est le dixième de celui observé sur le même bassin dénudé. Ces mêmes études prouvent que la production de sédiments à l'hectare peut être 70 fois plus faible dans le bassin boisé que dans le bassin dénudé (Arborescences, 1995).

- Un rôle de protection d'un milieu biologique particulièrement riche, présentant des espèces végétales et animales intéressantes, réunies dans des écotypes très particuliers. Ces milieux sont de plus très fragiles et leur retour à une situation d'équilibre y est lent (résilience faible ou nulle) en 
raison notamment des conditions bioclimatiques difficiles. Si un seuil d'irréversibilité biologique a été franchi, il ne peut y avoir de retour à moyen terme à l'écosystème initial sans intervention extérieure (Vallauri et Chauvin, 1997). C'est entre autres à cause de ce rôle de protection du milieu que l'on note logiquement la présence en zone de montagne en France d'une trentaine de réserves naturelles, de cinq parcs nationaux et d'une dizaine de parcs naturels régionaux.

- Enfin, un rôle récréatif et paysager sur des versants fortement exposés aux regards des populations montagnardes et de nombreux touristes (skieurs ou estivants). II suffit pour s'en convaincre de se rappeler l'émoi national provoqué par les conséquences de l'ouragan de 1982 à Chamonix.

Chacun de ces facteurs pris indépendamment des autres ne permet pas de définir une situation particulière à la montagne, mais leur conjonction peut induire un certain nombre de contraintes dans ces zones.

\section{Contraintes sylvicoles}

- L'enneigement prolongé fait peser une menace sur les régénérations et les peuplements adultes (avalanches, reptation du manteau neigeux, poids de la neige sur les branches, etc.) mais assure une couverture aux jeunes plants qui sont ainsi protégés des froids trop intenses. II diminue la période durant laquelle les interventions sont possibles, sachant qu'il existe d'autres contraintes. Le cumul de toutes ces contraintes rend parfois limitée voire inexistante la période durant laquelle l'intervention est possible.

- Les conditions climatiques et stationnelles réduisent la croissance des peuplements par rapport à la plaine entraînant normalement des durées de révolution allongées.

- L'hétérogénéité stationnelle impose, si le gestionnaire veut la prendre correctement en compte, une sylviculture fine, et nécessite donc de posséder un personnel nombreux et des outils performants: Système d'Informations géographiques (rôle de protection, étude paysagère, schéma de desserte, etc.), typologie des peuplements, catalogue des stations avec prise en compte de l'aspect dynamique, etc.

- Cependant, cette sylviculture se voit imposer de nombreuses obligations :

- rôle de protection ou paysager;

- quasi-impossibilité d'exploiter du bois d'industrie dont la mobilisation est souvent déjà non rentable en plaine, ce qui pose le problème de la non-réalisation des premières éclaircies ;

- gestion en futaie irrégulière qui a pour conséquences de priver de l'exonération trentenaire d'impôt foncier accordée aux plantations et d'engendrer une faible quantité de bois à récolter par hectare à chaque passage alors que des moyens spécifiques d'exploitation (câble, hélicoptère) nécessitent au contraire des prélèvements ponctuels denses ;

- problème de dépérissement dû à la pollution concentrée dans certaines vallées (trafic routier, industries lourdes, etc.) ;

- plantations non mécanisables à cause de la pente et des obstacles ;

- régénérations difficiles du fait de la pression des populations d'ongulés, ou plus rarement du pâturage, et des conditions édaphiques ou climatiques limitantes. La limite de régénération difficile est ainsi estimée à $1500 \mathrm{~m}$ en Savoie.

\section{Contraintes d'exploitation}

- La pente et l'existence de nombreux obstacles accroissent la pénibilité et les risques d'accidents du bûcheronnage (risques aggravés par l'éloignement des chantiers et leur mauvaise desserte), ce qui en augmente le coût. 
- C'est encore la pente qui limite le fonctionnement des machines de vidange des bois. Les ordres de grandeur suivants des limites imposées par la pente peuvent être retenus (Abeels, 1995) :

Tableau II

Engins de débardage permis suivant la pente

\begin{tabular}{|c|c|c|}
\hline Pente & Classe de pente & Équipement adapté \\
\hline 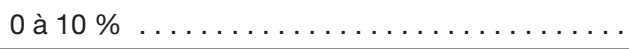 & De niveau & Tracteur agricole \\
\hline 10 à $30 \% \ldots \ldots \ldots \ldots \ldots \ldots \ldots \ldots \ldots$ & Légère & Porteur et débusqueur \\
\hline 30 à $60 \%$ & Prononcée & Débusqueur et/ou câble \\
\hline Plus de $60 \%$ & Escarpée & Câble ou hélicoptère \\
\hline
\end{tabular}

La présence d'obstacles peut de plus rendre toute utilisation d'engins impossible (présence de petites falaises par exemple).

- La pente renchérit le prix de la construction et de l'entretien des routes, des pistes et des places de dépôt. Un bilan récent des routes forestières dans les Pyrénées permet ainsi de dégager les trois principaux critères de coûts de ces routes qui sont dans l'ordre : la pente en travers, la proportion de rochers et le nombre d'ouvrages nécessaires (Auban, 1996 ; Auban et Bartoli, 1997). II convient de souligner que l'insuffisance de la desserte gêne non seulement l'exploitation, mais aussi l'ensemble des opérations sylvicoles : entretiens, inventaires, martelages, surveillance.

- L'état général du réseau routier rend le transport du bois plus long et plus difficile (voire impossible dans certains cas si on continue à exploiter en grandes longueurs), contrainte accrue par l'éloignement des centres de première transformation dû à la restructuration de l'aval de la filière ces dernières années.

- L'arrêt hivernal contraint les exploitants-scieurs à stocker trois à quatre mois de consommation à l'entrée de l'hiver : le coût financier de cette réserve est parfois très lourd à supporter et se répercute, à la baisse, sur le prix d'achat des bois. L'enneigement prolongé contraint à l'inactivité la maind'œuvre forestière et le matériel pendant une bonne partie de l'année. Ceci rend difficile le recrutement de cette main-d'œuvre à qui est proposé un travail saisonnier, pénible et qui demande une qualification certaine (utilisation d'une installation à câble, conduite d'engins dans la pente). Le matériel spécifique, qui est coûteux (câble, hélicoptère), devient plus difficile à amortir puisqu'il ne sert qu'une partie de l'année.

- L'utilisation d'un matériel spécifique oblige à avoir une organisation sans faille qui intègre toutes les étapes du martelage à la vidange, ce qui induit des coûts d'organisation.

- La récolte est très hétérogène avec, d'un côté, des bois présentant des défauts (bois mitraillés, fluorés ou pourris) et, de l'autre, des bois de très grande qualité (belles billes de Mélèze, de Pin cembro,...).

- Les conditions climatiques difficiles font qu'une grosse partie de la récolte peut provenir de chablis (leur part dans les récoltes augmente constamment depuis les années 50 ; dans les Alpes du Nord, elle est estimée actuellement à $30 \%$ ), qui provoquent :

- une perte de matière première due au bois cassé ou éclaté,

- une augmentation des coûts d'exploitation, car les tiges sont enchevêtrées et l'exploitation doit être rapide pour éviter la baisse de la qualité du bois,

- une chute des cours du bois par déséquilibre de l'offre en cas de gros accident, comme cela s'est vu en Savoie en 1990.

Tout ceci a pour conséquence finale de diminuer le revenu du propriétaire. 
En résumé, pour l'exploitation - mais beaucoup de facteurs restent valables pour les opérations sylvicoles - on peut reprendre le tableau suivant issu du Manuel d'exploitation forestière de l'ARMEF et du CTBA sur l'influence des différents facteurs (ARMEF, CTBA, 1994). En grisé sont portés les facteurs particulièrement importants en zone de montagne.

Tableau III $\quad$ Facteurs déterminant la productivité de l'exploitation forestière

\begin{tabular}{|c|c|c|}
\hline & Bûcheronnage manuel & Vidange \\
\hline \multicolumn{3}{|l|}{ Facteurs physiques } \\
\hline Terrain : obstacles, pente, portance, adhérence & $\bullet$ & $\bullet$ \\
\hline $\begin{array}{l}\text { Environnement : } \\
- \text { lignes EDF, téléphone, clôtures ........... }\end{array}$ & $\bullet \bullet$ & $\bullet$ \\
\hline $\begin{array}{l}\text { - périodes de chasse ou d'interdiction } \\
\text { (tourisme...) } \ldots \ldots \ldots \ldots \ldots \ldots \ldots \ldots \ldots \ldots\end{array}$ & - & - \\
\hline - passage chez un tiers & • & • \\
\hline Accessibilité de la coupe en voiture & $\bullet$ & $\bullet$ \\
\hline Climat : période de pluie ou de neige & $\bullet$ & $\bullet$ \\
\hline \multicolumn{3}{|l|}{ Facteurs forestiers } \\
\hline \multicolumn{3}{|l|}{ Peuplement : } \\
\hline - traitement, type de coupe, essences $\ldots . .$. & - & $\bullet$ \\
\hline - surface, volume total, volume arbre moyen .. & • & - \\
\hline - prélèvement, martelage, réserves/ha $\ldots \ldots$. & - & $\bullet$ \\
\hline - conformation des arbres $\ldots \ldots \ldots \ldots \ldots$ & $\bullet$ & $\bullet$ \\
\hline - propreté du sous-bois $\ldots \ldots \ldots \ldots \ldots \ldots$ & $\bullet$ & $\bullet$ \\
\hline $\begin{array}{l}\text { Circulations des machines (ou des câbles) : } \\
- \text { existence et état des pistes ou couloirs } \ldots \ldots \\
- \text { largeurs et distances à parcourir } \ldots \ldots \ldots \ldots \\
- \text { place de dépôt } \ldots \ldots \ldots \ldots \ldots \ldots \ldots \ldots\end{array}$ & & $\ddot{\bullet}$ \\
\hline $\begin{array}{l}\text { Contraintes sylvicoles : } \\
- \text { protection des semis } \ldots \ldots \ldots \ldots \ldots \ldots \ldots \\
- \text { traitement des rémanents, des souches } \ldots \ldots\end{array}$ & 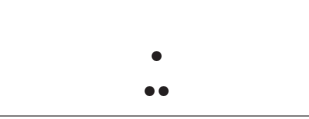 & $\bullet$ \\
\hline \multicolumn{3}{|l|}{ Facteurs relatifs au produit } \\
\hline Nombre de produits, longueurs, destination . & $\bullet$ & $\bullet$ \\
\hline Quantités/ha des différents produits $\ldots \ldots \ldots$. & & $\bullet$ \\
\hline Mode de réception $\ldots \ldots \ldots \ldots \ldots \ldots \ldots$ & $\bullet$ & • \\
\hline \multicolumn{3}{|l|}{ Facteurs relevant de l'organisation } \\
\hline Méthode employée (bois longs, bois courts, ...) & $\bullet$ & $\bullet$ \\
\hline Coordination bûcheronnage-vidange-transport & $\bullet$ & $\bullet$ \\
\hline Choix des moyens : personnels et machines ... & $\bullet$ & $\bullet$ \\
\hline Directives : sens de l'abattage orienté $\ldots \ldots \ldots$ & - & $\bullet$ \\
\hline - rangement des rémanents et produits & $\bullet$ & $\bullet$ \\
\hline - circulations à créer et/ou emprunter ........ & $\bullet$ & $\bullet$ \\
\hline Date et durée des activités $\ldots \ldots \ldots \ldots \ldots \ldots$ & - & $\bullet$ \\
\hline Contraintes réglementaires particulières $\ldots \ldots \ldots$ & $\bullet$ & $\bullet$ \\
\hline
\end{tabular}

$\bullet$ : facteur peu important.

- : facteur important.

•.• : facteur très important. 
Mais la forêt de montagne présente aussi des avantages qu'on ne peut négliger. On citera par exemple :

- la présence d'essences de valeur, à dominante plutôt résineuse, produisant des bois d'une qualité particulière grâce à des accroissements fins (qualités mécaniques, de résonance, esthétiques) avec comme exemple classique l'Épicéa de Haute-Savoie ;

- une capitalisation très marquée dans les massifs les moins bien desservis, ce qui peut favoriser des modes de vidange comme le câble ou l'hélicoptère ;

- une protection active ou passive contre les risques naturels à un coût modéré par rapport à des travaux de génie civil ;

- l'existence d'un patrimoine écologique et paysager qui peut être valorisé financièrement de façon directe ou indirecte (station de sport d'hiver, tourisme vert).

D'autre part, les contraintes pesant sur la forêt de montagne sont déjà, dans une certaine mesure, prises en compte avec:

- pour les communes forestières, un taux légal de frais de garderie versés à l'ONF modulé en faveur des zones de montagne (anciennement $8,5 \%$ contre $10 \%$ en plaine, actuellement $10 \%$ contre $12 \%)$;

- la possibilité de subventions à des taux importants, et même sans réel plafonnement, pour les travaux de construction de desserte;

- la possibilité pour les industries de première transformation de bénéficier de nombreuses sources de financement pour se moderniser : crédits FFN, prime d'orientation agricole secteur-bois, fonds d'intervention pour l'autodéveloppement en montagne (FIAM), fonds interministériel de développement et d'aménagement rural (FIDAR).

\section{L'EXEMPLE DE LA MAURIENNE (SAVOIE)}

\section{Une situation géographique et humaine définissant une identité forte}

La Maurienne correspond à la vallée de l'Arc et de ses affluents; elle s'étend sur $110 \mathrm{~km}$ d'Aiguebelle à Bonneval, de l'altitude 400 à $3181 \mathrm{~m}$ au Mont-Thabor. L'orientation générale de la vallée est Est-Ouest. Les sols sont variés (granites, schistes, calcaires, gypses) et les pentes souvent fortes.

Il existe soixante-trois communes en Maurienne; la sous-préfecture qui recouvre l'ensemble de la vallée est établie à Saint-Jean-de-Maurienne à $62 \mathrm{~km}$ de Chambéry, $110 \mathrm{~km}$ de Grenoble, $150 \mathrm{~km}$ de Lyon et $130 \mathrm{~km}$ de Turin. L'ONF y est organisé en deux divisions (Modane et Saint-Jean-deMaurienne) comptant chacune trois groupes techniques.

La population totale, 41207 habitants en 1990, est en légère baisse depuis le recensement de 1982. L'activité agricole ancienne a été supplantée depuis le début du siècle par l'industrie, attirée initialement par l'énergie hydroélectrique (aluminium, chimie). Le tourisme d'été et d'hiver s'est surtout développé depuis 1960 et est actuellement le principal secteur d'emploi (55\% dans le secteur tertiaire). L'emploi industriel est désormais en légère baisse.

La RN6 Paris-Lyon-Modane-Turin utilise la Maurienne et apporte un trafic soutenu avec I'Italie par le col du Mont-Cenis ou le tunnel de Fréjus-Modane. Elle est doublée par l'A86.

L'ensoleillement est important (300 jours/an à Valloire) et contrasté suivant les versants. L'exposition y est donc un facteur climatique prépondérant avec des "endroits" (adret) moins fortement boisés (pression anthropique) et présentant plus de feuillus que les "envers" (ubac) qui sont très frais. La température annuelle moyenne est basse $\left(5,3^{\circ} \mathrm{C}\right.$ à Lanslebourg), mais le réchauffement printanier 
est rapide. La pluviométrie varie de $550 \mathrm{~mm} / \mathrm{an}$ en fond de vallée à Avrieux à $1800 \mathrm{~mm}$ sur certains sommets de Basse-Maurienne, mais est généralement faible (zone intra-alpine sèche). L'enneigement dure de 4 à 6 mois suivant l'altitude. II convient enfin de signaler l'importance des vents, surtout "la bise" du nord mais aussi "le vent d'en bas" du sud-ouest et "la lombarde" venant de la vallée du Pô. Ceux-ci provoquent de forts chablis, comme en 1990 par exemple.

\section{Des peuplements forestiers homogènes}

Jusqu'en 1860, la Savoie faisait partie du Royaume sarde qui exploitait la forêt sans aménagement en fonction seulement de ses besoins. Depuis cette date, c'est-à-dire du rattachement de la Savoie à la France, la forêt a été prise en charge par l'administration française des Eaux et Forêts, qui a instauré des aménagements.

À partir de 1960, la Savoie a connu un développement important des stations de sports d'hiver, surtout en Tarentaise, mais aussi en Maurienne avec quatorze sites. Ce développement a eu des influences négatives sur les massifs forestiers (morcellement, défrichement).

En 1964, le Parc national de la Vanoise a été créé sur 53000 ha avec une zone périphérique de 144000 ha entre la Haute-Tarentaise et la Haute-Maurienne. Mais la zone centrale ayant été repoussée vers des altitudes élevées, le parc ne compte en fait que peu de peuplements forestiers.

Toutes ces évolutions favorisent un changement progressif de l'objectif de production qui n'est, malgré tout, pas abandonné, vers ceux d'accueil du public et de protection.

La forêt publique en Maurienne représente 30149 ha répartis en :

- 24115 ha $(80 \%)$ de forêts communales. En Maurienne, cinquante-huit communes sur soixante-trois sont propriétaires d'une forêt. Malgré sa relativement faible extension en surface, la forêt a donc un poids important dans la vie rurale.

- 6034 ha (20\%) de forêts domaniales, généralement très peu productives et correspondant à des terrains initialement non boisés acquis par l'État depuis un siècle afin de lutter contre la très forte érosion torrentielle. Environ 600 ha seulement de ces forêts sont à objectif de production de bois.

La forêt privée est peu importante en Moyenne et Haute-Maurienne, si l'on excepte les accrus récents sur les terrains agricoles abandonnés; elle est un peu plus importante en Basse-Maurienne. En tout, elle ne représente que 8300 ha très morcelés avec une moyenne de 2,3 ha par propriétaire.

L'Inventaire forestier national distingue deux régions forestières sur la vallée de la Maurienne (IFN, 1986) :

- la région Basse-Maurienne depuis Aiton jusqu'à La Chambre, regroupée dans les statistiques de l'IFN avec la Basse-Tarentaise et la vallée de La Rochette en raison de nombreuses similitudes. Le taux de boisement y est de $55,1 \%$;

- la région Maurienne depuis La Chambre jusqu'à Bonneval où le taux de boisement est de seulement $17,2 \%$.

$\mathrm{Au}$ total, le taux de boisement sur l'ensemble de la Maurienne est de l'ordre de $31 \%$ qu'on peut comparer aux taux de boisement national de 25,6\%, départemental de $28,5 \%$ et sur l'ensemble des sept grands massifs forestiers de montagne de $41 \%$. Pour une zone de montagne, cette superficie boisée est donc relativement faible en raison de l'importance des pelouses d'altitude et de l'activité humaine (installations industrielles, axes de communication) dans le fond de vallée. L'essence la plus représentée est l'Épicéa sur $40 \%$ de la surface, avec présence de Sapin jusqu'à $1100 \mathrm{~m}$, de Mélèze, Pin cembro ou Pin à crochets en altitude. En Basse-Maurienne, le feuillu, le Châtaignier principalement, peut devenir localement envahissant. 
L'IFN a défini pour la Savoie 11 types de peuplements. En Maurienne, les principaux types de peuplements et leurs caractéristiques sont les suivants :

Tableau IV

Les 4 principaux types de peuplements en Maurienne et leurs caractéristiques (IFN, 1986)

\begin{tabular}{|l|c|c|c|}
\hline & $\begin{array}{c}\text { \% de la surface } \\
\text { occupée }\end{array}$ & $\begin{array}{c}\text { Volume moyen } \\
\text { par hectare }\left(\mathrm{m}^{3} / \mathrm{ha}\right)\end{array}$ & $\begin{array}{c}\text { Accroissement } \\
\text { annuel moyen } \\
\left(\mathrm{m}^{3} / \mathrm{ha} / \mathrm{an}\right)\end{array}$ \\
\hline Pessière-sapinière $\ldots \ldots$ & 30,3 & 257 & 7,24 \\
\hline Mélèzin ............ & 17,2 & 217 & 5,15 \\
\hline Résineux d'altitude ..... & 15,7 & 211 & 6,23 \\
\hline Peuplement de pins ..... & 12,8 & 152 & 4,40 \\
\hline
\end{tabular}

Ces volumes à l'hectare sont importants par rapport au volume moyen à l'hectare national de $138 \mathrm{~m}^{3} / \mathrm{ha}$, et même de $174 \mathrm{~m}^{3} / \mathrm{ha}$ en ne considérant que les futaies. De plus, de même que les accroissements, ils ont tendance à augmenter. Cependant, il faut noter que la campagne de mesure de I'IFN a eu lieu en 1984 et que, depuis cette date, de nombreux peuplements du milieu de vallée ont été touchés par la pollution fluorée. Celle-ci a entraîné une forte mortalité et donc une baisse du volume sur pied. Les résultats de la troisième campagne d'inventaire en cours préciseront l'importance de cette baisse.

En raison des pentes fortes, l'accès à la forêt reste une difficulté essentielle sur pratiquement l'ensemble de la vallée. Malgré la construction de voies forestières, certaines zones restent inaccessibles aux camions grumiers et même aux tracteurs forestiers.

La pollution fluorée, qui affectait autrefois gravement 15000 ha de forêt de Saint-Étienne-de-Cuines à Modane, est en voie de disparition depuis 1987 : les rejets, provenant des usines Péchiney-UgineKuhlmann, sont en effet passés de 410 t/an de fluor en 1980 à 162 t/an en 1984 et 63 t/an depuis 1990.

Enfin, du fait du faible renouvellement des peuplements, il reste sur la Haute-Maurienne un certain nombre de peuplements mitraillés lors de la dernière guerre.

\section{La production du bois et la récolte}

La "production brute" moyenne évaluée par I'IFN est de $3,2 \mathrm{~m}^{3} / \mathrm{ha} / \mathrm{an}$ en Haute et MoyenneMaurienne et de $8,8 \mathrm{~m}^{3} / \mathrm{ha} / \mathrm{an}$ en Basse-Maurienne, en Basse-Tarentaise et dans la vallée de La Rochette.

En 1994, 82 exploitations forestières avaient leur siège social en Savoie, dont 41 n'étaient pas intégrées à une scierie. Pour une moyenne de plus de $40000 \mathrm{~m}^{3} / \mathrm{an}$ commercialisés, les exploitants de la vallée retirent $14000 \mathrm{~m}^{3} / \mathrm{an}$ et les scieurs de Maurienne travaillent $15000 \mathrm{~m}^{3} / \mathrm{an}$. La Maurienne ne dispose donc pas d'entreprises d'exploitation forestière ou de sciage susceptibles de prendre en charge la totalité des bois offerts. La récolte est constituée essentiellement de bois résineux : ces derniers représentent 90,5 \% du bois d'œuvre en 1994 en Savoie, et sont utilisés soit en sciage, soit en papeterie.

Selon l'ONF, en forêt publique de Maurienne, les prévisions de récoltes annuelles sont - sauf cataclysme naturel comme en 1983, 1984 et 1990 - de $44000 \mathrm{~m}^{3} / \mathrm{an}$, dont $2000 \mathrm{~m}^{3} /$ an feuillus, en volume tige, soit environ $50000 \mathrm{~m}^{3} / \mathrm{an}$ houppiers compris (figure 1, p. 169). 
Figure 1 VOLUME RÉCOLTÉ TOTAL EN FORÊTS PUBLIQUES EN MAURIENNE

(en $\mathrm{m}^{3}$ houppiers et écorce compris)

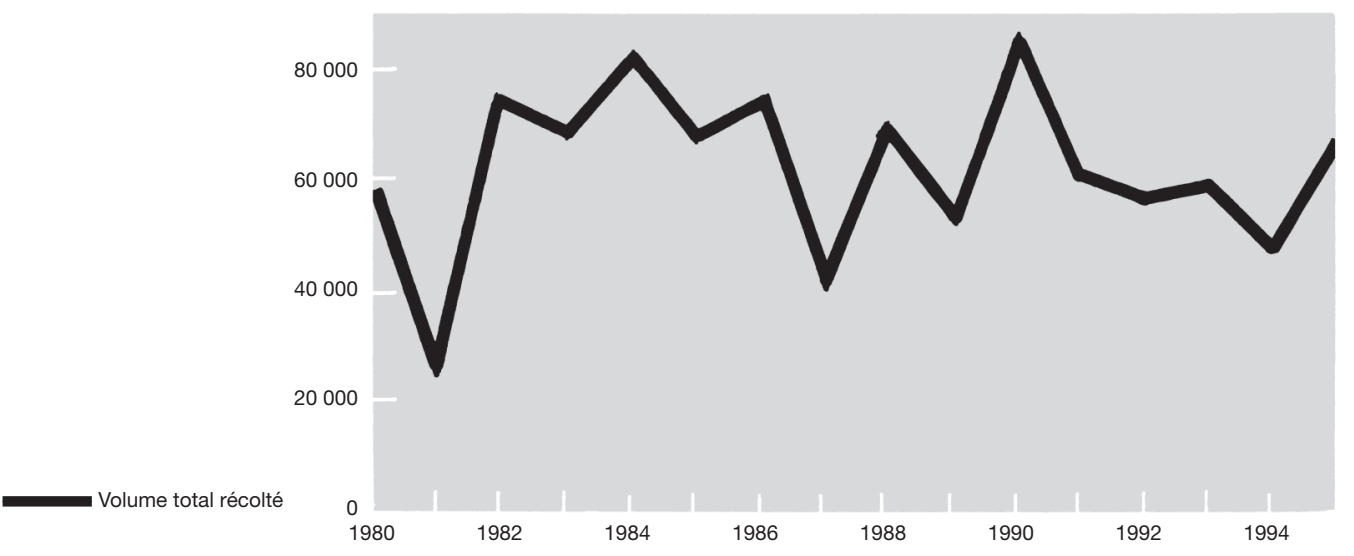

La Maurienne présente donc des caractéristiques propres à la forêt de montagne:

- La vallée est encaissée et son fond fortement occupé par l'urbanisation et une industrialisation ancienne ; les peuplements forestiers concentrés en deux étroites bandes de part et d'autre de l'Arc et de ses affluents. II existe donc presque uniquement des peuplements en situation de pentes fortes et continues qui rendent la gestion et l'exploitation forestières particulièrement difficiles.

- De plus, on se trouve dans les Alpes internes avec une pluviométrie faible $(500 \mathrm{~mm} / \mathrm{an}$ à Avrieux en fond de vallée), le Hêtre est donc absent et l'essence dominante est l'Épicéa (40\% de la surface) accompagné du Sapin jusqu'à $1100 \mathrm{~m}$, du Pin cembro ou du Pin à crochets plus haut. Les peuplements présentent en plus de cette homogénéité d'essence, une homogénéité de structure : ils ont généralement une structure irrégulière.

- La collecte des données pour l'exploitation forestière est facilitée par la décision de quelques communes forestières de Moyenne-Maurienne de développer l'exploitation en régie pour permettre la vente de bois façonnés et triés. Ce nouveau mode d'exploitation s'est accompagné de la mise en place d'un parc à grumes (capacité de $30000 \mathrm{~m}^{3}$ avec arrosage automatique, vente d'environ $15000 \mathrm{~m}^{3}$ par an) géré par l'ONF (groupe technique de Saint-Martin-la-Porte). Cette décision se justifie par l'importance des chablis sur la vallée et par la volonté de lutter contre l'augmentation des invendus apparue depuis le début des années 80 .

\section{HANDICAP ÉCONOMIQUE EN FORÊT DE MAURIENNE}

Quelles sont les conséquences économiques de ces contraintes, notamment sur l'exploitation forestière?

\section{Prix bois sur pied et pourcentage d'invendus}

Une donnée facilement accessible est le prix moyen des bois sur pied aux ventes d'automne de I'ONF. En théorie, le prix sur pied est le résultat de la soustraction au prix du bois façonné rendu scierie, des coûts techniques d'exploitation, à savoir coûts d'abattage et de vidange, et du coût du transport jusqu'à la scierie ainsi que des frais généraux de l'exploitant. L'observation de ces prix donne donc une idée de l'importance des coûts techniques d'exploitation. 
Les prix du Sapin et de l'Épicéa ont été comparés en Savoie et dans les principaux départements producteurs regroupés en deux groupes:

- trois départements aux conditions de montagne très marquées: Savoie, Haute-Savoie, Hautes-Pyrénées (pour ce dernier, pour le Sapin uniquement) ;

- les autres départements gros producteurs: Vosges, Doubs, Jura, Ain, Aude (pour ce dernier, pour le Sapin uniquement), Puy-de-Dôme. Ces départements témoins ne sont pas des départements "de plaine". Cependant, ils présentent des situations d'exploitation et de gestion forestières qui sont bien moins difficiles que dans les trois premiers départements.
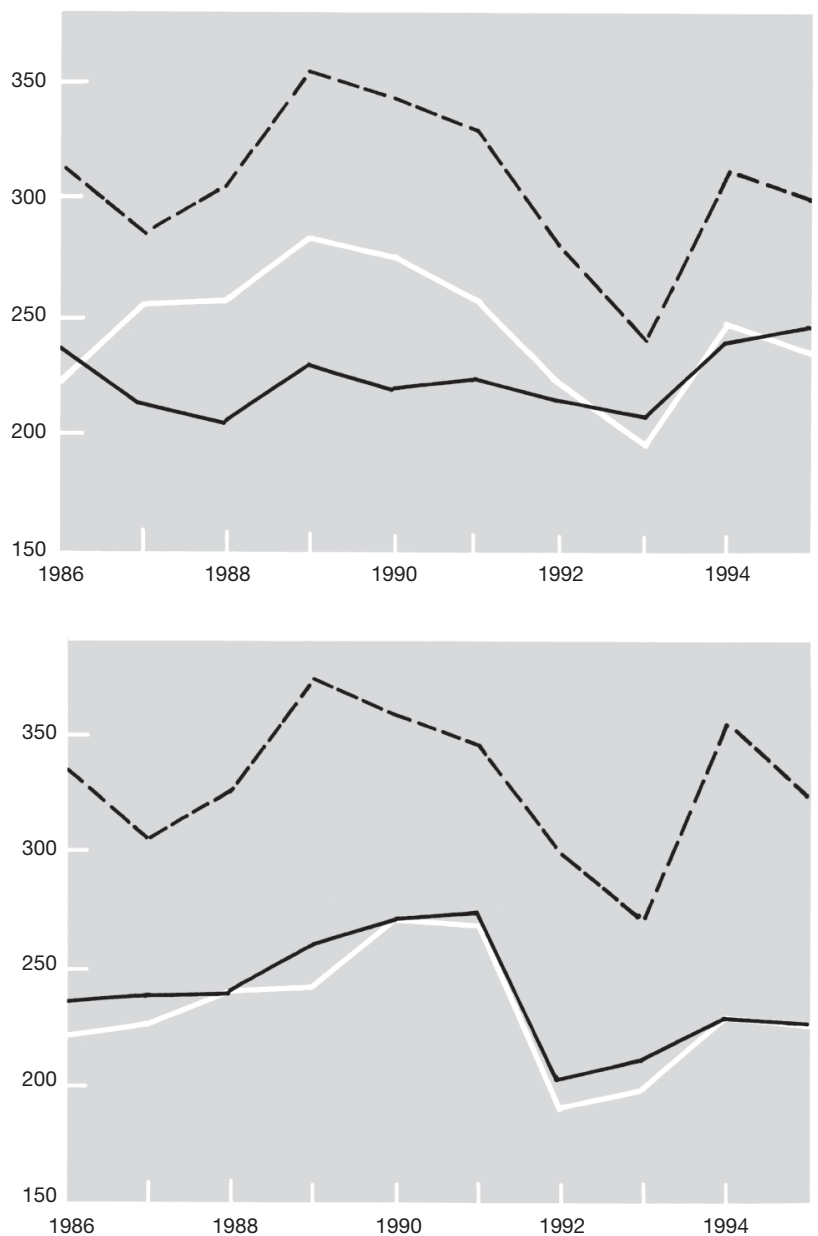

Figure 2

PRIX DU SAPIN EN SAVOIE, EN CONDITIONS DE MONTAGNE ET DANS LES DÉPARTEMENTS-TÉMOINS (en francs courants)

Figure 3 COMPARAISON DU PRIX DE L'ÉPICÉA EN SAVOIE, EN CONDITIONS DE MONTAGNE, ET DANS LES DÉPARTEMENTS-TÉMOINS (en francs courants)

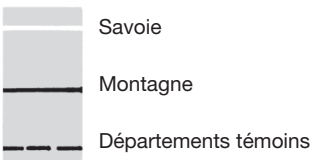

On constate une nette décote des prix sur pied pour les trois départements aux conditions de montagne difficiles. Elle est d'environ $80 \mathrm{~F}$ en moyenne sur la période 1986-1995 et peut monter certaines années à plus de $120 \mathrm{~F}$. Ceci reflète des problèmes d'organisation de la filière plus ou moins performante, de différence de qualité des bois (par exemple, la qualité du Sapin des Vosges ou de l'Aude est généralement très supérieure à la qualité du Sapin des Hautes-Pyrénées), etc. Cependant, la difficulté des conditions d'exploitation en montagne, qui en augmente le coût, reste l'un des principaux facteurs explicatifs. 
Les exploitants se tournant a priori d'abord vers les zones d'exploitation facile, en changeant de département au besoin, afin de garder la maîtrise de leurs coûts d'approvisionnement, les conditions d'exploitation difficiles devraient se traduire par de forts pourcentages d'invendus. Sur les trois départements Savoie, Haute-Savoie et Hautes-Pyrénées, ceux-ci sont généralement plus forts de vingt-cinq points par rapport aux départements témoins. Là encore, les difficultés d'exploitation ne sont pas le seul facteur explicatif, mais ils entrent assurément en ligne de compte.

Figure 4 POURCENTAGES D'INVENDUS EN MAURIENNE AUX VENTES DE BOIS SUR PIED (Source ONF)

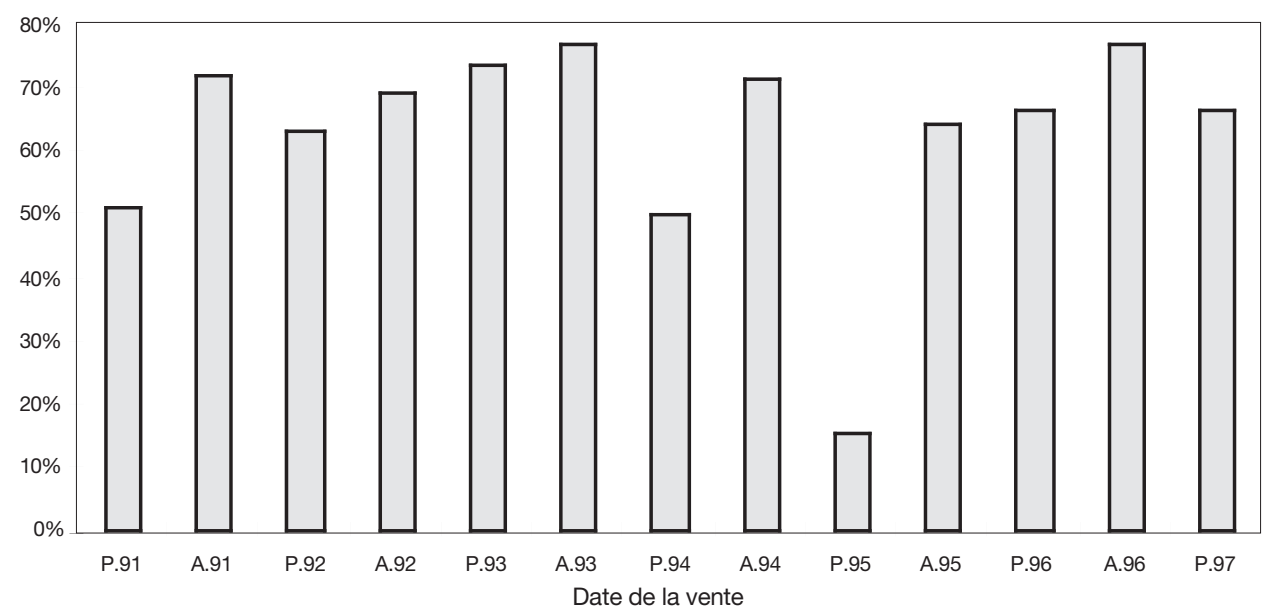

Pour les propriétaires, à une baisse des revenus induite par des prix d'achat plus faibles, s'ajoute donc une autre baisse des revenus du fait des invendus. De plus, la coupe étant aussi une opération sylvicole, particulièrement dans les peuplements irréguliers, ces invendus provoquent également un vieillissement, une perte de qualité, une plus grande fragilité au chablis pour le peuplement, qui nuisent là encore à sa valorisation future.

Ainsi, en région Rhône-Alpes, l'importance de ces invendus a entraîné un excédent de gros bois, avec un diamètre de plus de $60 \mathrm{~cm}$, représentant $20 \%$ du volume de bois régional, soit 8,5 millions de $\mathrm{m}^{3}$ situés essentiellement dans des zones d'accès difficile (ONF, Mission commerciale RhôneAlpes, 1995). Ces gros bois surannés voient souvent leur prix baisser du fait de la perte de qualité ou tout simplement de la difficulté à être déplacés étant donné leur poids.

\section{Évolution du SMIC par rapport au prix du bois en Savoie}

Parmi les facteurs entrant dans les coûts, il faut noter depuis les années 50 l'augmentation des charges, de l'énergie et des coûts de main-d'œuvre.

Pour cette dernière, il semble avéré que l'exploitation forestière et les opérations sylvicoles en zone de montagne aient beaucoup moins profité de la forte mécanisation qui a transformé les activités en plaine depuis les années 50. Des suivis de chantiers permettent d'estimer que, dans le prix de revient du câblage, le poste le plus important (60\%) est la main-d'œuvre (ONF, Mission commerciale Rhône-Alpes, 1995).

Toutes ces opérations étant donc restées plus manuelles, leur coût dépend toujours fortement du prix de la main-d'œuvre : il est par conséquent intéressant de comparer l'évolution du prix du bois et celle du SMIC horaire (figure 5, p. 172). 


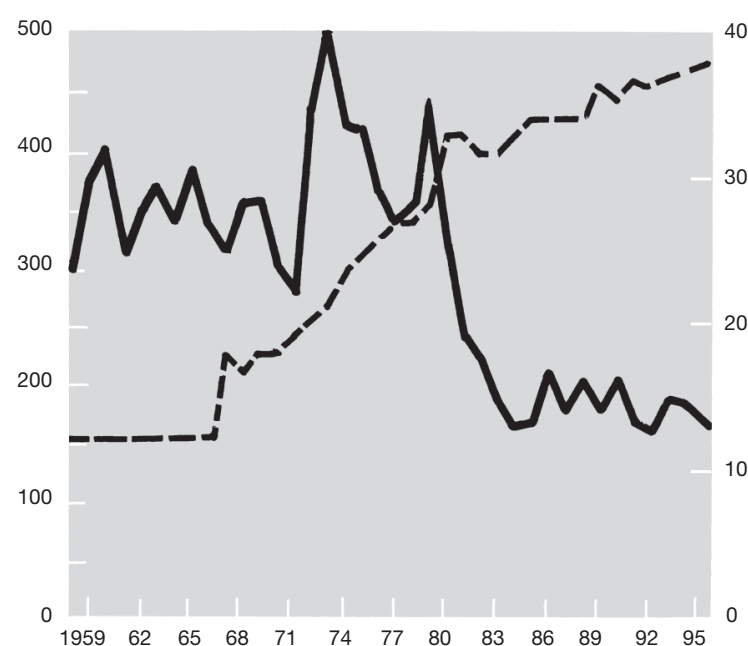

Figure 5

COMPARAISON DE L'ÉVOLUTION DU SMIC HORAIRE ET DU PRIX MOYEN DES BOIS SUR PIED EN SAVOIE EN FRANC 1996

(Source ONF et INSEE)

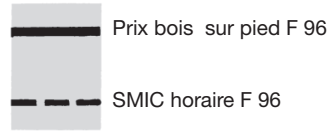

On peut ainsi distinguer trois périodes dans l'évolution du prix du bois sur pied : avant 1980, le prix oscille autour d'un niveau haut, dans les années 80 il baisse, puis se stabilise à un niveau bas. De même, pour le SMIC, il convient de différencier deux périodes : avant 1968, le SMIC horaire est fixé à un niveau bas (12 F 1996), et après 1968, il est régulièrement réévalué.

Dans ces conditions, le prix moyen $\mathrm{du}^{3} \mathrm{~m}^{3}$ de bois en heures de SMIC est passé en 40 ans de plus de 3 jours de travail à environ une demi-journée. Même en prenant en compte l'amélioration de la productivité qui s'est produite en parallèle, il devient de plus en plus difficile pour les propriétaires forestiers d'effectuer des travaux en les finançant uniquement à partir de la vente de bois. La solution peut passer en plaine par une mécanisation de plus en plus poussée, mais celle-ci n'est pas adaptée aux conditions de montagne du fait de la pente.

\section{Résultats du parc à grumes de Saint-Martin-la-Porte}

Le parc à grumes de Saint-Martin-la-Porte, qui regroupe des coupes provenant essentiellement des groupes techniques de l'ONF de Modane, Saint-Martin-la-Porte et Saint-Jean-de-Maurienne, fournit des données sur l'exploitation en Maurienne. En faisant le bilan des ventes de 1993 à 1996, on obtient les prix moyens des bois résineux façonnés. L'année 1993 est choisie comme année de départ bien que la vente de bois façonné ait commencé dès la fin des années 80 , car ce n'est qu'à partir de cette année-là qu'ont été mises en place définitivement les normes de classement, à savoir :

- Les catégories de diamètre au milieu correspondent à la norme AFNOR B53-300 de juillet 1991 (établi par le CTBA) :

\begin{tabular}{|ll|l|l}
- CL1 & $10-19 \mathrm{~cm}$ & - CL4 & $40-49 \mathrm{~cm}$ \\
- CL2 & $20-29 \mathrm{~cm}$ & - CL5 & $50-59 \mathrm{~cm}$ \\
- CL3 & $30-39 \mathrm{~cm}$ & - CL6 & $60-69 \mathrm{~cm}$
\end{tabular} \mid

- Lorsque les bois n'ont pas fait l'objet d'un classement normatif, ils sont notés NC pour non classés.

- Les Pins cembro et Mélèzes sont classés par référence aux habitudes locales. Ce classement n'a aucune relation avec le classement AFNOR :

- A : Menuiserie, charpente choisie ;

- B : Charpente courante ;

- D : Bardage. 
- Les Épicéas, Sapins et Pins (autres que cembro) sont classés suivant application locale de la norme AFNOR B53-300.

Tableau V

Application locale du classement AFNOR

\begin{tabular}{|c|c|c|c|c|c|}
\hline \multicolumn{2}{|c|}{ Qualité } & Nœud & $\begin{array}{l}\text { Classes } \\
\text { de diamètre } \\
\text { médian }\end{array}$ & $\begin{array}{l}\text { Diamètre } \\
\text { fin bout }\end{array}$ & $\begin{array}{l}\text { Singularités } \\
\text { et altérations } \\
\text { admises }\end{array}$ \\
\hline \multicolumn{2}{|c|}{ B } & $0-25 \mathrm{~mm}$ & Toutes & 25 & \\
\hline \multirow[t]{2}{*}{$\mathrm{B} / \mathrm{C}$} & $\mathrm{B} / \mathrm{C} 1$ & \multirow[t]{2}{*}{$25-40 \mathrm{~mm}$} & 3 et + & 20 & \\
\hline & $\mathrm{B} / \mathrm{C} 2$ & & 2 et - & 14 & \\
\hline \multirow[t]{2}{*}{ D } & D1 & \multirow[t]{2}{*}{$40 \mathrm{~mm}$ et +} & 3 et + & 14 & \multirow{2}{*}{$\begin{array}{l}\text { Fibre torse }>4 \mathrm{~cm} / \mathrm{m} \\
\text { Courbure simple }>2 \mathrm{~cm} / \mathrm{m} \\
\text { Courbure complexe }\end{array}$} \\
\hline & D2 & & 2 et - & 14 & \\
\hline \multicolumn{2}{|c|}{$\begin{array}{l}\text { Bois cubés } \\
\text { en stères } \\
\text { Billons } 2,05 \\
\text { et } 2,50 \mathrm{~m} \\
\text { Palette }\end{array}$} & $40 \mathrm{~mm}$ et + & - & 14 & $\begin{array}{l}\text { Roulure } \\
\text { Échauffure dure } \\
\text { Petits trous } \\
\text { Surface carbonisée } \\
\text { Arbre sec }\end{array}$ \\
\hline
\end{tabular}

À Saint-Martin-la-Porte, sur les quatre années étudiées, on obtient les prix et volumes suivants :

Tableau VI

Prix des bois résineux façonnés au parc à grumes (moyenne 1993-1996)

\begin{tabular}{|c|c|c|c|}
\hline Essences & Qualité & 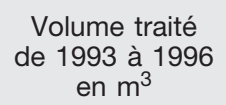 & $\begin{array}{l}\text { Prix moyen } \\
\text { en } \mathrm{F} 1996 / \mathrm{m}^{3}\end{array}$ \\
\hline \multirow[t]{5}{*}{ Sapin-Épicéa } & $\mathrm{NC}$ & 9401 & 370 \\
\hline & Palette & 2867 & 268 \\
\hline & D2 & 7189 & 341 \\
\hline & D1 & 14586 & 440 \\
\hline & D & 7422 & 344 \\
\hline \multirow[t]{5}{*}{ Pin sylvestre } & $\mathrm{B} / \mathrm{C} 2$ & 721 & 334 \\
\hline & $\mathrm{B} / \mathrm{C} 1$ & 6155 & 506 \\
\hline & $\mathrm{B} / \mathrm{C}$ & 646 & 442 \\
\hline & B & 351 & 527 \\
\hline & Total & 49338 & 395 \\
\hline \multirow[t]{5}{*}{ Mélèze } & $\mathrm{NC}$ & 228 & 474 \\
\hline & $\mathrm{D}$ & 568 & 491 \\
\hline & B & 1325 & 653 \\
\hline & A & 447 & 1032 \\
\hline & Total & 2568 & 667 \\
\hline Pin cembro & & 369 & 650 \\
\hline Total résineux & & 52475 & 410 \\
\hline
\end{tabular}


Ces prix sont un point de référence pour estimer les coûts techniques d'exploitation et de transport également relevés sur le parc à grumes. Pour une coupe moyenne, ceux-ci ne doivent pas dépasser $410 \mathrm{~F} 1996 / \mathrm{m}^{3}$ sous peine de voir la coupe déficitaire. Ce chiffre ne doit cependant pas masquer la grande hétérogénéité des prix pouvant varier de 1 à 4 .

Or, sur un ensemble de 88 coupes (76 coupes effectuées au tracteur divisées en deux classes de difficulté définie suivant le montant des coûts techniques d'exploitation, 4 coupes effectuées à l'aide d'un câble, 8 coupes effectuées à l'aide d'un hélicoptère) relevées de 1987 à 1997, les coûts techniques d'exploitation suivants ont pu être définis :

Tableau VII Coûts techniques d'exploitation relevés au parc à grumes de Saint-Martin-la-Porte

\begin{tabular}{|l|c|c|c|c|}
\hline Type de coupe & Abattage & Vidange & Transport & Total \\
\hline Coupe au tracteur "facile" & 66 & 67 & 49 & 182 \\
\hline Coupe au tracteur "difficile" . & 79 & 98 & 49 & 226 \\
\hline Coupe au câble .......... & 101 & 149 & 49 & 299 \\
\hline Coupe à l'hélicoptère ....... & 90 & 297 & 49 & 436 \\
\hline
\end{tabular}

Dans les coûts techniques d'exploitation, on a distingué :

- le coût d'abattage ;

- le coût de vidange (tracteur, câble ou hélicoptère) ;

- le coût de transport depuis la coupe jusqu'au parc à grumes, qu'on a fixé à la moyenne pour l'ensemble des chantiers de 1993 à 1997 , soit $49 \mathrm{~F} / \mathrm{m}^{3}$.

Même si ces moyennes ont été effectuées sur un petit nombre de coupes prises dans le même secteur, elles donnent une bonne estimation de coûts techniques d'exploitation en conditions difficiles.

Pour qu'un chantier difficile par hélicoptère ne soit pas déficitaire, il est absolument nécessaire que l'exploitant soit assuré que les bois lui soient payés au moins $440 \mathrm{~F} 1996 / \mathrm{m}^{3}$. De même, des coupes avec une exploitation facile au tracteur risquent de se révéler déficitaires si elles ne produisent que du bois de très faible qualité d'une valeur inférieure à $180 \mathrm{~F} 1996 / \mathrm{m}^{3}$. On retrouve là l'impossibilité relevée en conditions de montagne d'exploiter valablement du bois d'industrie.

En fait, ce sont surtout les coûts de vidange qui évoluent. En effet, alors que les coûts d'abattage évoluent dans un rapport de 1 à 2,4 , les coûts de vidange évoluent dans un rapport de 1 à 7,8 . C'est donc essentiellement le niveau de ces derniers qui va décider de la rentabilité ou non de la coupe.

Finalement, la différence moyenne sur les coûts d'abattage et de vidange entre des coupes faciles

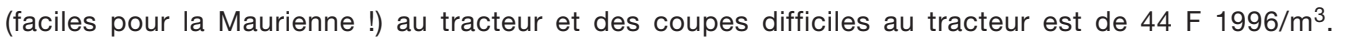
Ceci peut en première analyse être relevé comme mesure du "handicap" montagne.

\section{Comparaison avec des chantiers d'exploitation dans le Doubs}

Depuis trois ans, l'ONF dans le Doubs effectue également des coupes en régie d'entreprise dans des peuplements de résineux avec vidange au tracteur. Le Doubs n'est pas à proprement parler un "département de plaine". Mais il présente des peuplements résineux et des conditions d'exploitation moins difficiles : topographie plus douce, pas d'enjeu de RTM. II peut servir ici de référence. 
Figure 6 COÛTS D'ABATTAGE ET DE VIDANGE EN FRANCS 1996 EN MAURIENNE, DANS LE DOUBS ET SUR 3 CHANTIERS ÉTUDIÉS

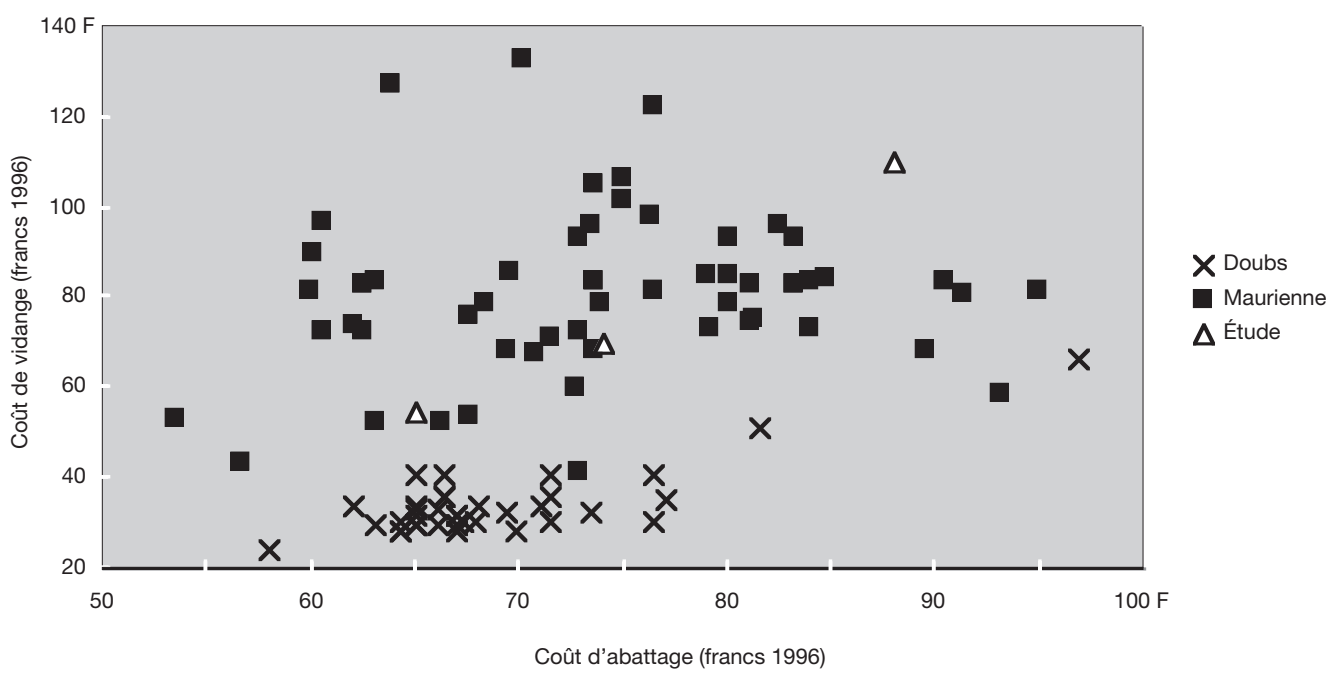

Les coûts d'abattage sont comparables dans les deux zones avec une moyenne de $70 \mathrm{~F} / \mathrm{m}^{3}$ et une fourchette allant de $50 \mathrm{~F} / \mathrm{m}^{3}$ à $100 \mathrm{~F} / \mathrm{m}^{3}$. Mais elles se distinguent par les coûts de vidange. Pour le Doubs, la moyenne des coûts de vidange est de $34 \mathrm{~F} / \mathrm{m}^{3}$ avec une fourchette entre 24 et $66 \mathrm{~F} / \mathrm{m}^{3}$, alors qu'en Maurienne, la moyenne est de $82 \mathrm{~F} / \mathrm{m}^{3}$, avec une fourchette entre 42 et $133 \mathrm{~F} / \mathrm{m}^{3}$. On obtient un surcoût moyen de la montagne de $48 \mathrm{~F} / \mathrm{m}^{3}$ dû aux difficultés de vidange. Cette évaluation s'avère donc très proche de la précédente.

\section{CONCLUSION ET PERSPECTIVES}

Pour résumer, on peut dire que dans le cas de la Maurienne:

- Le coût d'abattage est assez semblable entre la "plaine" et la "montagne". Ceci n'est pas étonnant puisqu'on a comparé des chantiers à abattage non mécanisé. La principale difficulté pour l'abattage en montagne est bien souvent la difficulté d'accès à la coupe, du fait du mauvais réseau de desserte.

- Le coût de vidange au tracteur augmente très vite avec les difficultés liées à la montagne : le surcoût est en moyenne de $50 \mathrm{~F}$ 1996/m³. L'exploitation en montagne applique bien souvent sans adaptation des méthodes de vidange conçues et optimisées pour des situations de "plaine".

- Lorsque la vidange a lieu au câble ou à l'hélicoptère, les coûts explosent et deviennent difficilement comparables avec ceux de "plaine" (surcoûts respectifs de 120 et 260 F 1996/m³).

- Enfin, une contrainte supplémentaire des régions de montagne consiste dans le transport des grumes jusqu'à un réseau routier correct.

Ces observations nécessitent de mettre en lumière deux limites:

- Tout d'abord, le montant des surcoûts de cet handicap ne doit pas être généralisé à l'ensemble des forêts de montagne puisque, comme l'ont montré Barthod et Pignard, les situations rencontrées sont diverses. Sans même quitter la Savoie, il n'est pas difficile de trouver des forêts de plateau présentant peu de difficultés d'exploitation. 
- Ensuite, il faut être conscient que les surcoûts estimés le sont dans l'état actuel des techniques et des méthodes employées pour l'exploitation en Maurienne. II existe d'importants gisements de progrès, comme une meilleure concertation entre tous les acteurs de la filière, une meilleure adaptation mutuelle de l'exploitation et de la sylviculture, une coordination au sein des massifs pour assurer du travail aux câblistes et inversement assurer aux coupes à câble d'être exploitées, la conception de schémas de desserte intégrés, etc. Tant que ces techniques et ces méthodes n'auront pas été optimisées, il peut paraître inefficace de tenter de remédier au "handicap montagne" par une seule subvention.

Le parallèle avec le Doubs permet de mettre en évidence le handicap technique des bois de montagne au niveau de la mobilisation. II ne préjuge pas de la qualité technologique, souvent appréciée en montagne. II n'empêche que les problèmes d'accessibilité pénalisent la valorisation première et, concrètement, la rémunération du propriétaire.

Une conséquence directe de ce handicap technique est la déprise sylvicole qui se manifeste maintenant clairement dans de nombreux massifs forestiers de montagne. Cette déprise, qui a d'abord touché les zones les moins productives comme les Alpes du Sud, est maintenant également effective dans les zones les plus difficiles des Alpes du Nord. Ainsi, une étude du CEMAGREF sur 6900 ha de forêts ayant un rôle de protection dans le secteur de Moutiers (Tarentaise) montre que $67 \%$ d'entre elles ne sont plus exploitées, que $22 \%$ connaissent des coupes épisodiques et que $11 \%$ seulement sont encore exploitées régulièrement.

À gauche, vue du parc à grumes de Saint-Martin-la-Porte.

À droite, façonnage en forêt communale de Saint-Julien-Montdenis : les difficultés du travail du bûcheron entraîne des risques pour ce dernier et des dégâts au peuplement.
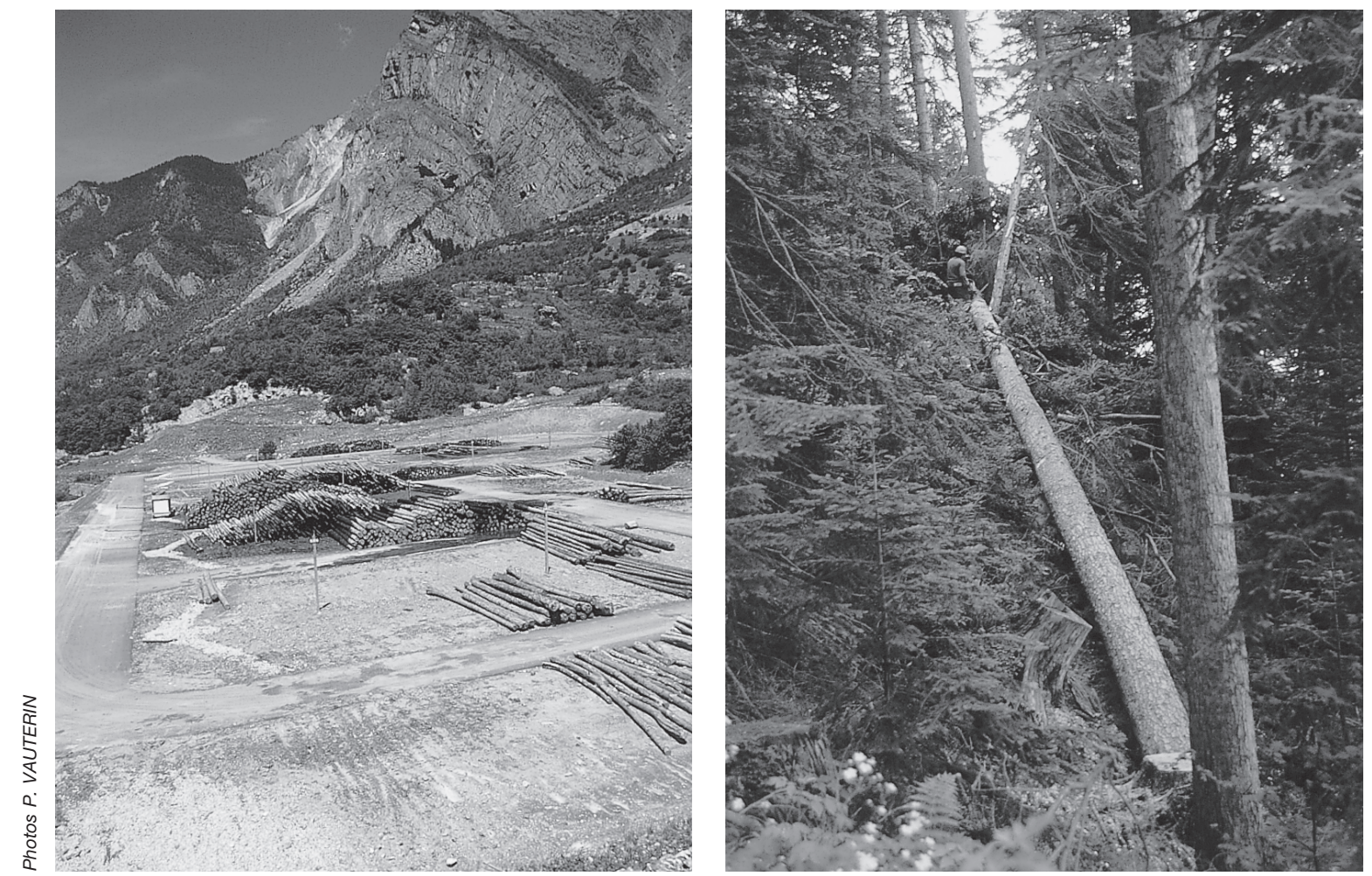
Cela dit, à l'accessibilité des parcelles, et malgré les évolutions des techniques et des infrastructures, viennent s'ajouter des paramètres qui procèdent des autres fonctions de la forêt de montagne, généralement d'ordre social, qui lui confèrent un rôle d'utilité publique, maintes fois reconnu au niveau des instances nationales et surtout internationales. À la fonction de protection, reconnue depuis la fin du siècle dernier avec l'avènement des services de restauration des terrains en montagne, sont venues s'ajouter les utilités environnementales (sites botaniques, zoologiques). Dans ce cadre, il est intéressant de constater l'évolution du type de propriété en fonction de l'altitude : la propriété privée est généralement située sur le piémont, la forêt communale lui succède et les forêts d'altitude sont souvent propriétés de l'État. À cet égard, cette classification grossière, mais néanmoins pertinente, fait ressortir le caractère d' "utilité publique" des forêts de montagne.

Le gestionnaire et l'ensemble des opérateurs de la filière-bois doivent désormais, dans les zones qui cumulent les différents handicaps, définir sinon des arbitrages, au moins des hiérarchisations entre les différentes fonctions. Une des illustrations de ces arbitrages est la "saisonalisation" des fonctions: l'été pour les promeneurs, l'automne pour les chasseurs, l'hiver pour les skieurs et le printemps pour la reproduction des animaux. Cette caricature peut dans certains cas devenir réalité et pose le problème de la planification et de la réalisation des opérations de gestion forestière (dont l'exploitation forestière fait partie intégrante) quelles qu'elles soient.

En fait, l'enjeu actuel est de pouvoir valoriser la gestion patrimoniale des forêts de montagne par la seule fonction économique. II n'est effectivement actuellement pas concevable, sociologiquement

Débardage au câble en forêt communale d'Albiez-le-Jeune.

À gauche, installation du treuil Wyssen.

À droite, descente d'une grume.
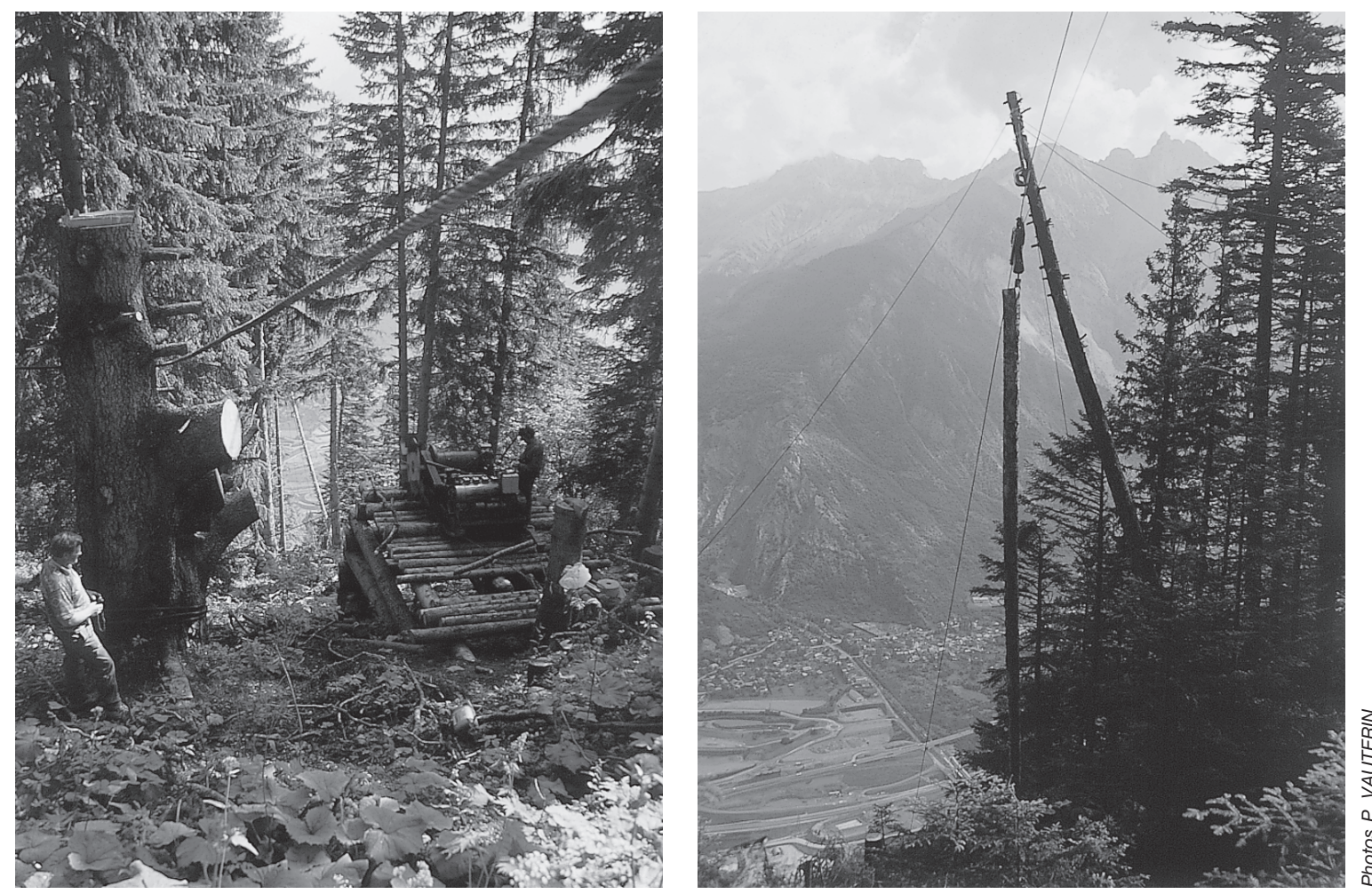
parlant, d'instaurer des péages pour l'accès aux forêts françaises (alors qu'il est déjà bien difficile de faire respecter les interdictions sur les routes et pistes forestières), même si cela ne choque pas de payer à l'entrée des grands parcs nationaux de certains pays.

Par contre, il convient de prendre en compte les bénéfices apportés par la présence de ces forêts de montagne. Ainsi, en plaine, la valeur du service rendu par certaines forêts qui protègent des nappes phréatiques aux abords d'agglomérations importantes est déjà estimée dans certains cas. De même, en montagne, il serait par exemple bon d'être conscient du prix de la protection physique apportée en beaucoup d'endroits par la forêt, prix qui pourrait être comparé à celui de travaux de génie civil apportant la même protection.

Cette multifonctionnalité des forêts de montagne existe depuis toujours ; elle est aujourd'hui exacerbée en tant que contrainte à cause des difficultés économiques de la filière-bois. Or, pour garantir la santé, l'attrait et la richesse de ce patrimoine, dans la majorité des cas, la gestion forestière participait directement au financement de ces fonctions et au maintien de ce capital social. Le problème réside dans le fait que la filière économique est de plus en plus déficiente dans les zones véritablement de montagne où les bois sont de moindre qualité ou par trop inaccessibles. Dans ce schéma, il convient de trouver des alternatives économiques susceptibles de maintenir, où cela est nécessaire, une activité minimale afin de préserver ce capital et ceux qui en vivent directement ou indirectement. II est effectivement regrettable que ce caractère multifonctionnel soit parfois perçu par les acteurs de la filière-bois comme un handicap, alors qu'il pourrait être un atout.

II ne faut en effet pas tomber dans le catastrophisme: des initiatives sont développées et la manière de considérer la situation a bien évolué. Ces réactions, ces évolutions sont également riches d'enseignements voire exemplaires pour l'ensemble de la filière-bois en montagne et ailleurs en plaine. Elles exacerbent effectivement des problèmes beaucoup plus diffus ailleurs. À ce titre, elles peuvent être considérées comme un laboratoire de ce que pourraient être les relations entre les différents "partenaires" de la filière : la multifonctionnalité suggère la concertation, la décision partenariale et l'élaboration de schémas alternatifs qui peuvent également être riches d'enseignement pour l'ensemble du territoire. À ce titre, on peut même parler d' "atout montagne" plutôt que de "handicap montagne". En effet, quand les schémas classiques ne fonctionnent plus, cela pousse à développer des voies alternatives nouvelles et qui enrichissent (dans tous les sens du terme si possible) l'ensemble de ceux qui y participent. Ainsi, jusqu'à aujourd'hui, les acteurs de la forêt de montagne ont lutté pour la reconnaissance de l'“exception montagnarde", c'est-à-dire la reconnaissance de l'identité montagnarde afin de faire adapter les règlements et les interventions publiques aux conditions spécifiques locales. Désormais, il conviendrait de faire reconnaître l'"excellence montagnarde", c'est-à-dire de porter la filière forêt-bois en montagne à un très haut niveau de qualité.
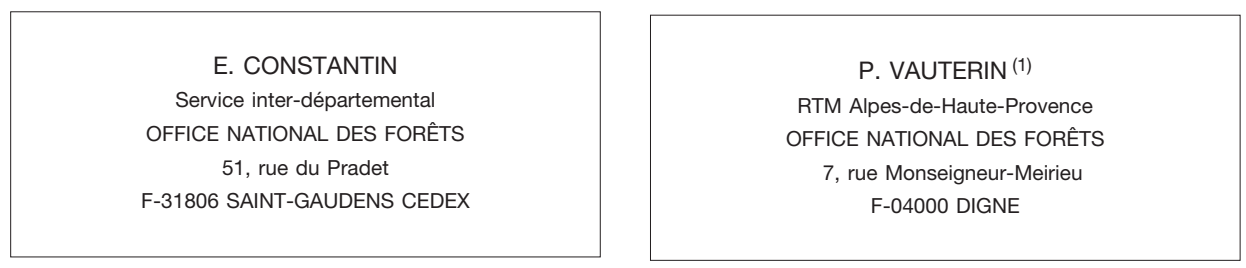

(1) Ancien stagiaire ONF - STIR Alpes. 


\section{BIBLIOGRAPHIE}

ABEELS (P.). - Génie forestier, technologies et techniques en exploitation forestière et travaux forestiers. - Paris : Masson, 1995. - pp. 92-94.

ARMEF, CTBA, MSA. - Manuel d'exploitation forestière. Tome II. - 1994. - pp. 249-250.

AUBAN (J.-M.). - Trente ans de routes forestières dans les Pyrénées. - Nancy: ENGREF, 1996. - 71 p. + annexes (Mémoire de $3^{\mathrm{e}}$ année de la FIF).

AUBAN (J.-M.), BARTOLI (M.). - Les Routes forestières de montagne. Bilan et propositions techniques. L'exemple des Pyrénées centrales. - Revue forestière française, vol. XLIX, n 3, 1997, pp. 235-246.

BARTHOD (C.), PIGNARD (G.). - De la forêt de montagne... aux forêts en montagne. - Aménagement et Nature, $\mathrm{n}^{\circ} 120$, hiver 1995-96, pp. 41-54.

Gérer la forêt de montagne. - Arborescences, n 55, mars-avril 1995, pp. 3-18.

INVENTAIRE FORESTIER NATIONAL. - Département de la Savoie. Résultats de l'inventaire forestier (1974-1975). - IFN, 1975.

INVENTAIRE FORESTIER NATIONAL. - Département de la Savoie. Résultats du deuxième inventaire forestier (19851986). - Tomes 1 et 2. - IFN, 1986.

ONF, Mission commerciale Rhône-Alpes. - Rapport de synthèse du groupe câble. - 1995. - 76 p.

RAMEAU (J.-C.), MANSION (D.), DUMÉ (G.). - Flore forestière française. Guide écologique illustré. Tome 2 : Montagnes. - Paris : Institut pour le Développement forestier, 1993. - pp. 17-26.

SERVICE CENTRAL D'ENQUÊTES ET DE STATISTIQUES AGRICOLES (SCESA). - Enquête annuelle de branche "Scierie-Exploitation forestière". - annuel.

VALLAURI (D.), CHAUVIN (Ch.). - L'Écologie de la restauration appliquée à la forêt. - Revue forestière française, vol. XLIX, n³, 1997, pp. 195-203.

VAUTERIN (P.). - Le Handicap montagne et ses incidences sur l'exploitation forestière: le cas de la Maurienne. Nancy : ENGREF, 1997. - 77 p. (Rapport de stage de fin d'études).

LE HANDICAP MONTAGNE ET SES INCIDENCES SUR LA GESTION ET L'EXPLOITATION FORESTIÈRES : LE CAS DE LA MAURIENNE (SAVOIE, FRANCE) (Résumé)

La forêt en montagne connaît un certain nombre de contraintes techniques du fait même de sa situation. Les acteurs de la filière parlent du "handicap montagne". L'étude dégage quelles sont les contraintes qui pèsent sur l'exploitation et la gestion forestières en montagne, et montre comment sur une zone précise d'étude, la Maurienne, elles peuvent effectivement définir un handicap économique, qui est mesuré. On invite à repenser ce handicap dans le cadre de la multifonctionnalité des forêts de montagne.

THE MOUNTAIN DISADVANTAGE AND ITS IMPACT ON LOGGING AND FOREST MANAGEMENT : THE CASE OF THE MAURIENNE FOREST (SAVOIE, FRANCE) (Abstract)

Mountain forests are dominated by number of technical constraints arising from the location itself. Forestry professionals refer to this a the "mountain handicap". This study identifies the constraints for felling and forest management in mountain areas and show how, in the Maurienne area under study, they are indeed a measurable economic disadvantage. This disadvantage is then reconstrued in the perspective of the multifunctional character of mountain forests. 